\title{
A Guide to Companies
}

Absolute Clarity \& Calibration, LLC

Advanced Analysis Technologies, LLC.

Advanced Diamond Technologies

Advanced MicroBeam, Inc.

Advanced Microscopy Techniques

Advanced Research Corporation

Advance Surface Microscopy

AFMWorkshop

AIST-NT

Alliance Technologies

Amerinex Applied Imaging, Inc.

Amptek, Inc.

Anasys Instruments

Anatech USA

Anderson Materials Evaluation

Andor Technology

Angstrom Scientific Inc.

A.P.E. Research SRL

AppFive, LLC

Applied Beams LLC

Applied Electro-Optics Inc.

APPLIED IMAGE Inc.

Applied Physics Technologies, Inc.

Applied Precision, a GE Healthcare Company

Applied Spectra, Inc.

Applied Thermal Control

ASI/Applied Scientific Instrumentation

ASM International

ASPEX Corporation

Asylum Research

Attocube Systems AG

AttoLight AG

Aurora Spectral Technologies

Axiom Optics

B \& W Tek, Inc.

Basler Inc.

Bel-Art Products

BIGC: Dino-Lite Scopes

Bioptechs

BioTools

Bitplane/Andor Technology

Black Mesa Imaging, LLC

Boeckeler Instruments Inc. www.absoluteclarity.com

www.advancedanalysistech.com

www.thindiamond.com

www.advancedmicrobeam.com

www.amtimaging.com

www.arcnano.com

www.asmicro.com

www.afmworkshop.com

www.aist-nt.com

www.alliancetechgroup.com

www.amerineximaging.com

www.amptek.com

p. 17

www.anasysinstruments.com

www.anatechusa.com

www.andersonmaterials.com

www.andor.com

www.angstrom.us

www.aperesearch.com

www.appfive.com

www.appliedbeams.com

p. 3

www.aeousa.com

www.appliedimage.com

www.a-p-tech.com

p. 4

www.alliedprecision.com

www.appliedspectra.com

www.app-therm.com

www.asiimaging.com

p. 5

www.asminternational.org

www.aspexcorp.com

www.asylumresearch.com

www.attocube.com

www.attolight.com

www.auroraspectral.com

www.axiomoptics.com

www.bwtek.com

www.baslerweb.com/microscopy

p. 17

www.belart.com

www.bigc.com

www.bioptechs.com

www.btools.com

www.bitplane.com

http://blackmesa-imaging.com

www.boeckeler.com 


\section{Company Index}

\section{A Guide to Companies}

BrandTech Scientific, Inc.

Bruker AXS Inc.

Bruker MicroCT

Bruker Nano Surfaces

Bruker Optics

Buehler

CAMCOR

CAMECA

Capovani Brothers Inc.

Carbon Design Innovations

Carestream Molecular Imaging

CeramOptec

Charles Supper Company Incorporated

Chemlmage Corporation

Chroma Technology Corp.

Cobolt AB

ColdEdge Technologies

CoolLED Limited

CPGlobalTech

CRAIC Technologies

CrEST srl

CrISEL Instruments S.R.L.

CRYO Industries of America, Inc.

Dage-MTI

Datacolor, Inc.

Daylight Solutions, Inc.

Deben

Delaware Diamond Knives, Inc.

Delong America Inc.

Denton Vacuum, LLC

DiATOME U.S.

DiCon Fiberoptics, Inc.

DigisENS

Direct Electron, LP

Dune Sciences, Inc.

Duniway Stockroom Corp.

Ealing Catalog

E. A. Fischione Instruments, Inc.

EDAX, Inc.

Electron Microscopy Sciences

Electro-Optics Technology, Inc.

Electron Optica, Inc.

Energy Beam Sciences, Inc. www.brandtech.com

www.bruker-axs.com

www.bruker-microct.com

www.bruker.com/AFM

p. 17

www.bruker.com

www.buehler.com

www.camcor.uoregon.edu

www.cameca.com

www.capovani.com

www.cdi-nano.com

www.carestreamhealth.com

www.ceramoptec.com

www.charles-supper.com

www.chemimage.com

www.chroma.com

www.cobolt.se

www.coldedgetech.com

www.coolled.com

p. 17

www.cpglobaltech.com

www.microspectra.com

www.crestopt.com

www.crisel-instruments.it

www.cryoindustries.com

www.dagemti.com

www.scientific.datacolor.com

www.daylightsolutions.com

www.deben.co.uk

p. 17

www.ddk.com

www.lv-em.com

www.dentonvacuum.com

www.emsdiasum.com

pp. 6,18

www.diconfiberoptics.com

www.digisens.fr

www.directelectron.com

www.dunesciences.com

www.duniway.com

www.ealingcatalog.com

www.fischione.com

www.edax.com

p. 18

www.emsdiasum.com

www.eotech.com

www.electronoptica.com

www.ebsstore.com 


\section{Company Index}

\section{A Guide to Companies}

ETS-Lindgren

Evactron by XEI Scientific

Evans Analytical Group

Evex Analytical

Excelitas Technologies (X-Cite)

EXpressLO LLC

Exprodo Software

FEI Company

Fischione Instruments

Fluid Imaging Technologies, Inc.

Gamma Vacuum

Gatan, Inc.

Geller MicroÅnalytical Laboratory, Inc.

Glas-Col, LLC

Glen Mills Inc.

Globe Scientific Inc.

Hamamatsu Corporation

Harrick Scientific Products, Inc.

Haydon Kerk Motion Solutions, Inc.

Heidenhain Corporation

HEMCO Corporation

Herzan LLC

Hiden Analytical Limited

Hirox-USA, Inc.

Hitachi High Technologies America, Inc.

Hooke College of Applied Sciences

HORIBA Scientific

HREM Research, Inc.

Hummingbird Scientific

Hysitron, Inc.

ibidi, LLC

ibss Group, Inc.

iLab Solutions

Imina Technologies

Integrated Dynamics Engineering IDE

International Centre for Diffraction Data (ICDD)

ION-TOF

Iridian Spectral Technologies

IXRF Systems, Inc.

Janis Research Co.

Jasco

JENOPTIK Optical Systems GmbH

JEOL USA, Inc. www.ets-lindgren.com

www.evactron.com

www.eag.com

www.evex.com

www.excelitas.com

p. 18

www.EXpressLO.com

p. 18

www.expodo.com

www.fei.com

www.fischione.com

www.fluidimaging.com

www.gammavacuum.com

www.gatan.com/MT

p. 8

www.gellermicro.com

www.glascol.com

www.glenmills.com

www.globescientific.com

www.hamamatsu.com

p. 18

www.harricksci.com

www.haydonkerk.com

www.heidenhain.com

www.hemcocorp.com

www.herzan.com

www.hidenanalytical.com

www.hirox-usa.com

www.hitachi-hightech.com/us

www.hookecollege.com

www.horiba.com/scientific

www.hremresearch.com

www.hummingbirdscientific.com

www.hysitron.com

p. 19

www.ibidi.com

www.ibssgroup.com

p. 19

www.ilabsolutions.com

www.imina.ch

www.ideworld.com

www.icdd.com

p. 19

www.iontof.com

www.iridian.ca

www.ixrfsystems.com

p. 19

www.janis.com

www.jascoinc.com

www.jenoptik.com/progres

p. 19 


\section{Company Index}

\section{A Guide to Companies}

JPK Instruments AG

Kett US

Keyence Corporation of America

KeySight Technologies (Formerly Agilent Tech.)

Kimball Physics, Inc.

KonTEM GmbH

Kratos Analytical Inc.

K-Tek Nanotechnology

Kurt J. Lesker Company

L.A. Giannuzzi \& Associates LLC

Ladd Research Industries

Lambert Instruments

Laserglow Technologies

LatticeGear, LLC

LaVision BioTec

LECO Corporation

Lehigh Microscopy School

Leica Microsystems, Inc.

LeRoy Eyring Center for Solid State Science

Linkam Scientific Instruments

Lumencor, Inc.

Lumen Dynamics

Lumenera Corporation

LW Scientific

Lyncee Tec

Lytron

Mad City Labs, Inc.

MAX Detector Repair Group LLC

Max Levy Autograph

McCrone Associates

McCrone Atlas of Microscopic Particles

McCrone Microscopes \& Accessories

McCrone Research Institute

Meadowlark Optics

Media Cybernetics

Meiji Techno America

M.E. Taylor Engineering, Inc.

Micro Photonics

Micro Star Technologies Inc.

Microscopy Innovations, LLC

Microscopy/Microscopy Education (MME)

Microtechnics

Microtome Service Company www.jpk.com

www.kett.com

www.keyence.com/microscopes

p. 9

www.keysight.com/find/nano

www.kimballphysics.com

www.kontem.de

www.KRATOS.com

www.kteknano.com

www.lesker.com

www.LAGiannuzzi.com

www.laddresearch.com

www.lambertinstruments.com

www.laserglow.com

www.latticegear.com

www.lavisionbiotec.com

www.leco.com

www.lehigh.edu/microscopy

www.leica-microsystems.com

le-csss.asu.edu

www.linkam.co.uk

pp. 19,20

www.lumencor.com

www.ldgi-xcite.com

www.lumenera.com

www.Iwscientific.com

www.nanoandmore.com

www.lytron.com

www.madcitylabs.com

www.maxdetector.com

www.maxlevy.com

www.mccroneassociates.com

www.mccroneatlas.com

www.mccronemicroscopes.com

www.mcri.org

www.meadowlark.com

p. 20

www.mediacy.com

www.meijitechno.com

www.semsupplies.com

www.microphotonics.com

www.microstartech.com

www.microscopyinnovations.com

www.MicroscopyEducation.com

www.microtechnics.com

www.microtomeserviceco.com p. 20 


\section{Company Index}

\section{A Guide to Companies}

Microwave Research and Applications, Inc.

Microyn Techologies, Inc.

Minitool Inc.

Minus K Technology, Inc.

Mitutoyo America Corp.

Modern Microscopy

Motic Instruments Inc

Moxtek, Inc.

MTI Instruments/Fullam

NanoAndMore USA, Inc.

Nanofactory Instruments AB

Nanolane

Nanomechanics, Inc.

NanoMEGAS

Nanonics Imaging Ltd.

Nanoprobes Incorporated

Nanoscience Instruments

Nanosurf AG

Navitar

Nea Spec GmbH

Nightsea

Nion Co.

Norcada, Inc.

Norsam Technologies, Inc.

nPoint, Inc.

NT-MDT America Inc.

Object Research Systems

Olympus America, Inc.

Olympus Corporation of the Americas Scientific Solutions Group

Olympus Soft Imaging Solutions GmbH

Omniprobe, Inc.

optek-Danulat, Inc.

Optics Balzers

OptoTech, Inc.

Optronics

Oregon Physics

Oxford Instruments America, Inc.

Oxford Instruments NanoAnalysis USA

Park Systems, Inc.

Particle Technology Labs

PCO AG

Pemtron Corporation www.microwaveresearch.com

www.microyntech.com

www.minitoolinc.com

p. 20

www.minusk.com

www.mitutoyo.com

www.modernmicroscopy.com

www.motic.com

www.moxtek.com

www.mtiinstruments.com

p. 20

www.nanoandmore.com

www.nanofactory.com

www.nano-lane.com

www.nanomechanicsinc.com

www.nanomegasusa.com

www.nanonics.co.il

www.nanoprobes.com

www.nanoscience.com

www.nanosurf.com

www.navitar.com

p. 21

www.neaspec.com

www.nightsea.com

www.nion.com

www.norcada.com

www.norsam.com

www.npoint.com

www.ntmdt.com

www.theobjects.com

www.olympusamerica.com

www.olympus-lifescience.com

p. 21

www.soft-imaging.net

www.omniprobe.com

www.optek.com

www.opticsbalzers.com

www.optotech.com

www.optronics.com

www.oregon-physics.com

p. 21

www.oxford-instruments.com

www.oxinst.com/nanoanalysis

www.parkafm.com

www.particletechlabs.com

www.pco-tech.com

www.pemtron.com 


\section{Company Index}

\section{A Guide to Companies}

Pfeiffer Vacuum

Phenom-World

Photometrics

Photon etc.

Photonics Media/Laurin Publishing

PHOTONIS

Physical Electronics, Inc.

PI (Physik Instrumente)

PicoQuant GmbH

Piezosystem Jena Inc.

PNDetector

PNSensor GmbH

Polylnsight LLC

Princeton Instruments

Prizmatix

PRO Scientific

Protochips

PulseTor LLC

QImaging

Quantom Design, Inc.

Quartz Imaging Corporation

Quorum Technologies, Ltd.

Raith America, Inc.

Raptor Photonics

Rayspec Ltd.

Renishaw, Inc.

ResAlta Research Technologies Corp.

Rigaku Corporation

Rigaku Raman Technologies

RMC Products/Boeckeler Instruments

Royal Microscopical Society

RT Instruments, Inc.

SAES Getters USA, Inc.

SCHOTT North America, Inc. - Lighting and Imaging

Scientific Instruments \& Applications, Inc.

SCI Institute

SemionCompany

SEMTech Solutions, Inc.

Seron Technologies Inc.

SII Nanotechnology USA, Inc.

SimPore/TEMWindows

Siskiyou Corporation

SmarAct Inc. www.pfeiffer-vacuum.com

www.phenom-world.com

p. 10

www.photometrics.com

www.photonetc.com

www.photonics.com

www.xscellcamera.com

www.phi.com

www.pi-usa.us/

www.picoquant.com

www.piezosystem.com

p. 11

www.pndetector.de

p. 12

www.pnsensor.de

www.polyinsight.com

www.princetoninstruments.com

www.prizmatix.com

www.proscientific.com

www.protochips.com

p. 13

www.pulsetor.com

www.qimaging.com

www.qdusa.com

www.qrtz.com

www.quorumtech.com

www.raith.com

www.raptorphotonics.com

www.rayspec.co.uk

www.renishaw.com

www.resaltatech.com

www.rigaku.com

p. 21

www.rigakuraman.com

www.rmcproducts.com

www.rms.org.uk

www.rtinstruments.com

www.saesgetters.com

www.us.schott.com/lightingimaging

p. 21

www.sia-cam.com

www.sci.utah.edu

www.semionco.com

www.semtechsolutions.com

www.serontech.co.kr

www.siintusa.com

www.temwindows.com

www.siskiyou.com

www.smaract.com 


\section{Company Index}

\section{A Guide to Companies}

Smart Imaging Technologies

South Bay Technology, Inc.

Special Optics

SPECS Surface Nano Analysis GmbH

Spectronics Corporation

SPI Supplies

Spicer Consulting Limited

SPOT Imaging Solutions

SputterEtch Tech, Inc. Dba: Anatech USA

Stanford Photonics, Inc.

STAR Cryoelectronics/H.K.N. Inc.

Struers Inc.

Sutter Instrument

Synergy Vacuum, Inc.

Technical Manufacturing Corporation

tec5usa

Ted Pella, Inc.

TEMWindows.com (SimPore Inc.)

Tescan USA, Inc.

TGS Technologies, LLC.

The Microscope

Thermo Fisher Scientific

TILL Photonics GmbH - an FEI Company

Toshiba Imaging Systems Division

Tousimis Research Corporation

TREK, INC.

TVIPS GmbH

Ushio America, Inc.

UVP LLC

Visage Imaging

VSG, Visualization Sciences Group, Inc.

Vutara Super-Resolution Microscopy

Wafer World, Inc

Well Diamond Wire Saws, Inc.

WITec Instruments Corp. GmbH

XEI Scientific, Inc.

\section{ZEISS}

Zemax, LLC

Zemetrics a Zygo Company

Zeta Instruments

Zumatrix, Inc.

Zygo Corporation www.simagis.com

www.southbaytech.com

www.specialoptics.com

p. 22

www.specs.com

www.spectroline.com

www.2spi.com

p. 22

www.spicerconsulting.com

p. 22

www.spotimaging.com

www.anatechusa.com

www.stanfordphotonics.com

www.starcryo.com

www.struers.com

www.sutter.com

p. 22

www.synergyvacuum.com

www.techmfg.com

www.tec5usa.com

www.tedpella.com

pp. 22,23

www.temwindows.com

www.tescan-usa.com

www.tgstechnologies.net

www.mcri.org

www.thermoscientific.com

www.till-photonics.com

www.toshibacameras.com

www.tousimis.com

www.trekinc.com

www.tvips.com

www.ushio.com

p. 23

www.uvp.com

www.visageimaging.com

www.vsg3d.com

www.vutara.com

www.waferworld.com

p. 14

www.welldiamondwiresaws.com

www.witec.de

(see Evactron by XEI Scientific)

www.zeiss.com/microscopy

www.zemax.com

p. 15

www.zemetrics.com

www.zeta.inst.com

www.zumatrix.com

www.zygo.com 